\title{
Catecholamine Detection Using a Functionalized Poly(L-dopa)- Coated Gate Field-Effect Transistor
}

\author{
Taira Kajisa, ${ }^{* \dagger}$ †⿺ Wei Li, ${ }^{\ddagger}$ and Tsuyoshi Michinobu ${ }^{\ddagger}$ \\ ${ }^{\dagger}$ PROVIGATE Inc., The University of Tokyo Entrepreneur Plaza, 7-3-1 Hongo, Bunkyo-ku, Tokyo 113-0033, Japan \\ ${ }^{\ddagger}$ Department of Materials Science and Engineering, Tokyo Institute of Technology, 2-12-1 Ookayama, Meguro-ku, Tokyo 152-8552, \\ Japan
}

Supporting Information

ABSTRACT: A highly sensitive catecholamine (CA) sensor was created using a biointerface layer composed of a biopolymer and a potentiometric detection device. For the detection of CAs, 3-aminophenylboronic acid (3$\mathrm{NH}_{2}$-PBA) was reacted with the carboxyl side chain of L-3,4-dihydroxyphenylalanine (L-dopa, LD) and the PBA-modified L-dopa was directly copolymerized with $\mathrm{LD}$ on an Au electrode, resulting in a $3.5 \mathrm{~nm}$ thick PBAmodified poly(PBA-LD/LD) layer-coated Au electrode. By connecting the PBA-LD-coated $\mathrm{Au}$ electrode to a field-effect transistor (FET), the molecular charge changes at the biointerface of the Au electrode, which was caused by di-ester binding of the PBA-CA complex, were transduced into gate surface potential changes. Effective CAs included LD, dopamine (DA), norepinephrine $(\mathrm{NE})$, and epinephrine (EP). The surface potential of the PBA-LD-coated $\mathrm{Au}$ changed after the addition of $40 \mathrm{nM}$ of each CA

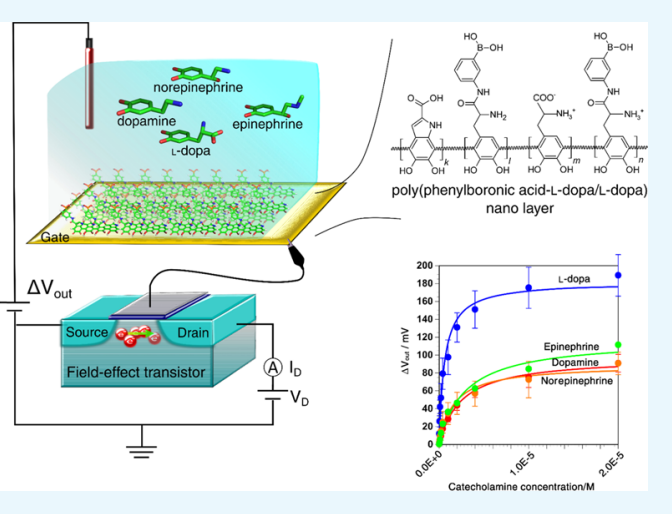
solution; notably, the PBA-LD-coated Au showed a higher sensitivity to LD because the surface potential change could already be observed after $1 \mathrm{nM}$ of LD was added. The fundamental parameter analyses of the PBA-LD to CA affinity from the surface potential shift against each CA concentration indicated the highest affinity to $\mathrm{LD}$ (binding constant $\left(K_{\mathrm{s}}\right): 1.68 \times 10^{6} \mathrm{M}^{-1}$, maximum surface potential shift $\left(V_{\max }\right): 182 \mathrm{mV}$ ). Moreover, the limit of detection for each CA was $3.5 \mathrm{nM}$ in LD, $12.0 \mathrm{nM}$ in DA, $7.5 \mathrm{nM}$ in NE, and $12.6 \mathrm{nM}$ in EP. From these results, it is concluded that the poly(PBA-LD/LD)-coated gate FET could become a useful biosensor for neurotransmitters, hormones, and early detection of Parkinson's disease.

\section{INTRODUCTION}

Catecholamines (CAs) play an important role in functional regulation of the body by acting as hormones and neurotransmitters. Among the CAs, dopamine (DA), norepinephrine (NE), and epinephrine (EP) act as adrenal medullary hormones and are responsible for sympathetic nerve action. Thus, it has been reported that the concentration of these three CAs in the blood increases in response to tension and stress. ${ }^{1}$ Furthermore, CAs have been measured as adrenal tumor biomarkers because it was reported that the total blood CA concentration is about 10 times higher in the case of adrenal medullary pheochromocytoma development than a few $\mathrm{nM}$ value normally found in the blood. ${ }^{2,3}$ Additionally, L-dopa (LD), which is the starting material for all CA metabolism and derived from tyrosine, is used in anti-Parkinson's disease medication because it can cross the blood-brain barrier. ${ }^{4,5}$ Because CA is successively metabolized in short order by enzymes from $\mathrm{LD}$ to $\mathrm{EP}$, it is necessary to detect the total CA levels in body fluids. ${ }^{6}$ However, CAs have been measured mainly by fractionation and quantification using high-performance liquid chromatography, an easy-to-use and direct detection method for CA level measurements that is expected to become accessible for point-of-care testing.
CAs have another unique characteristic. They can undergo oxidative self-polymerization to form polycatecholamines, which are biomimetic materials that are similar to the adhesive substance secreted from mussels. ${ }^{10}$ From the first report of polycatecholamine, its application has been studied because it could adhere to a wide variety of materials including polymers and metals. ${ }^{9,11-14}$ We focused on the chemical, adhesive, and easy-to-synthesize properties of polycatecholamine and applied them to create an electrode interface for biosensing.

The field-effect transistor (FET) biosensor is based on the principle of detecting charges of biomolecules at the gate electrode interface and has been developed to obtain information from ionic substances in a nonlabeling, rapid, and quantitative method. ${ }^{15-17}$ Thus, FETs have been successful in measuring the charge changes of ions, DNA, saccharides, proteins, and living cells adjacent to the gate surface in a highly sensitive and quantitative manner. ${ }^{18-22}$ An extended-gate FET, which contains a sensing gate electrode separated from the detection portion, is a useful tool for the

Received: March 19, 2018

Accepted: June 8, 2018

Published: June 20, 2018 
Scheme 1. Device Schematic, Chemical Structures, and Mechanisms of the CA FET Biosensor ${ }^{a}$

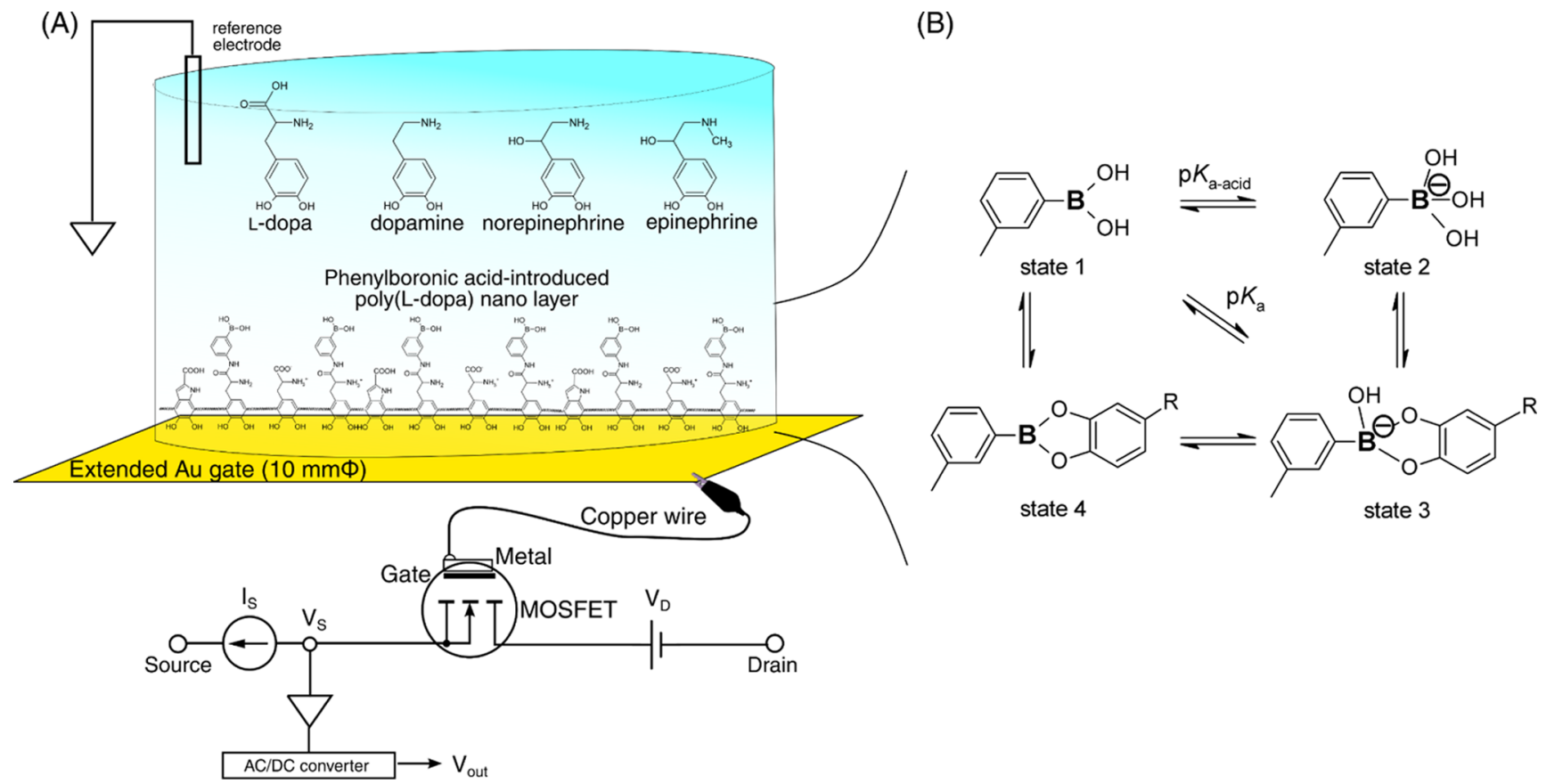

${ }^{a}$ (A) Schematic diagram of PBA-containing poly(LD) nanolayer [poly(PBA-LD/LD)]-coated gate FET. The Au gate electrode was connected to an extended metal-oxide semiconductor field-effect transistor (MOSFET). (B) Equilibrium of PBA with CAs containing a diol at the interface of the Au gate electrode.

construction of FET biosensors because we can design the material and shape of the gate electrode freely so as to match the interfacial modifications with the properties of the targeted molecules. ${ }^{23}$ Various saccharides have been reportedly quantified from as few as tens of $\mu \mathrm{M}$ using an extended-gate FET whose $\mathrm{Au}$ gate electrode was modified with a phenylboronic acid self-assembled monolayer (PBA-SAM). ${ }^{24}$ The boron atom in the PBA molecule changes from a neutral to a negative state when bound to vicinal diol compounds, such as sugars, through an equilibrium reaction so that the PBA-SAMcoated gate FET can capture the charge on the boron atom generated at the gold electrode interface in response to changes in sugar concentration. In the present study, as shown in Scheme 1, we constructed a thin layer of PBA-containing poly(L-dopa) on a gold electrode utilizing the unique characteristics of L-dopa, which polymerizes through a selfoxidization process and strongly adheres to metals. By efficiently capturing the charge events at the interface of an FET (Scheme 1A), we aimed to selectively detect CAs that possess diol structures based on the PBA equilibrium mechanism (Scheme 1B).

\section{RESULTS AND DISCUSSION}

Organic Synthesis of the PBA-LD Monomer and Electropolymerized Formation of Poly(PBA-LD/LD) on an Au Surface. To introduce a PBA molecule onto LD as a monomer, an amide formation reaction between the amino group of $\mathrm{NH}_{2}$-PBA and the carboxyl group of the LD side chain was utilized in the presence of the dehydration reagent 4(4,6-dimethoxy-1,3,5-triazin-2-yl)-4-methylmorpholinium chloride (DMT-MM) (Scheme 2). The synthesized product was identified by ${ }^{1} \mathrm{H}$ NMR, as shown in Figure 1 . The ${ }^{1} \mathrm{H}$ NMR indicated that the resulting product is the mixture of the designed product $\mathrm{PBA}-\mathrm{LD}$ and reagent $\mathrm{NH}_{2}$-PBA. The
Scheme 2. Synthetic Pathway of Poly(PBA-LD/LD)
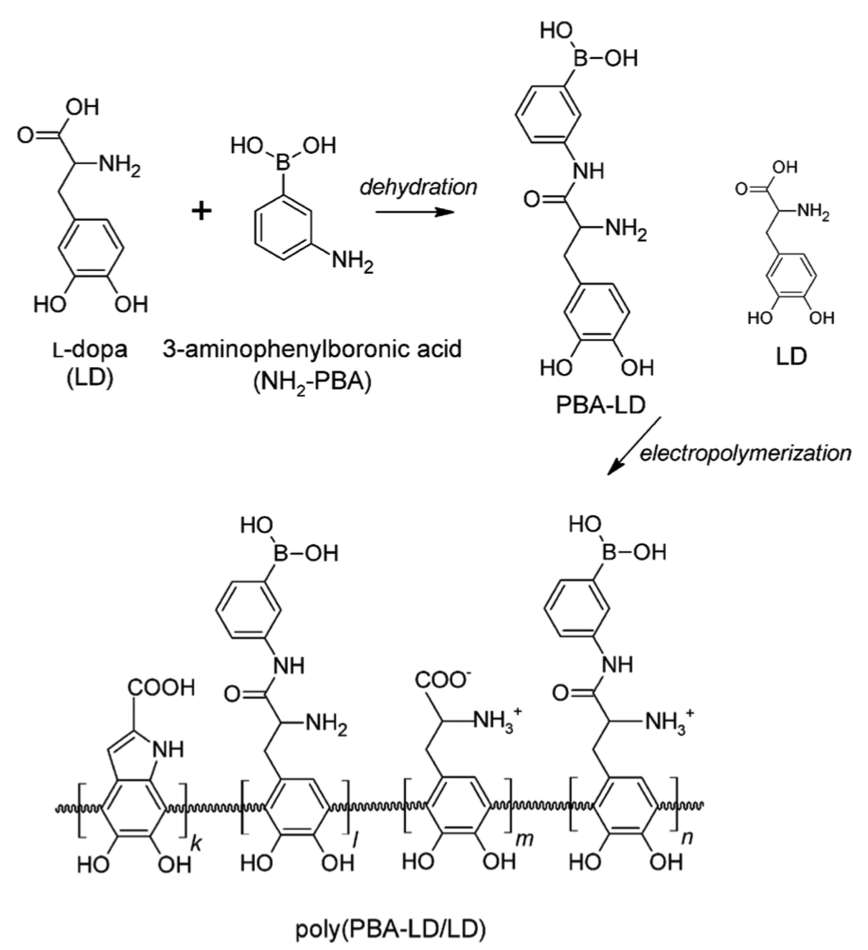

hydrogen peaks in PBA-LD (Figure $1 \mathrm{~b}, \mathrm{c}$ ) appeared after 15 min reaction and gradually increased over $4 \mathrm{~h}$, whereas the hydrogen peaks in $\mathrm{NH}_{2}$-PBA (Figure 1a) decreased. The conversion efficiency was calculated from the peak area ratio of the $\mathrm{NH}_{2}$-PBA hydrogen (Figure 1a) and the PBA-LD hydrogen (Figure $1 \mathrm{~b}, \mathrm{c}$ ). The ratio of unreacted $\mathrm{NH}_{2}-\mathrm{PBA}$ and synthesized PBA-LD in the solution was 3:2, indicating a 

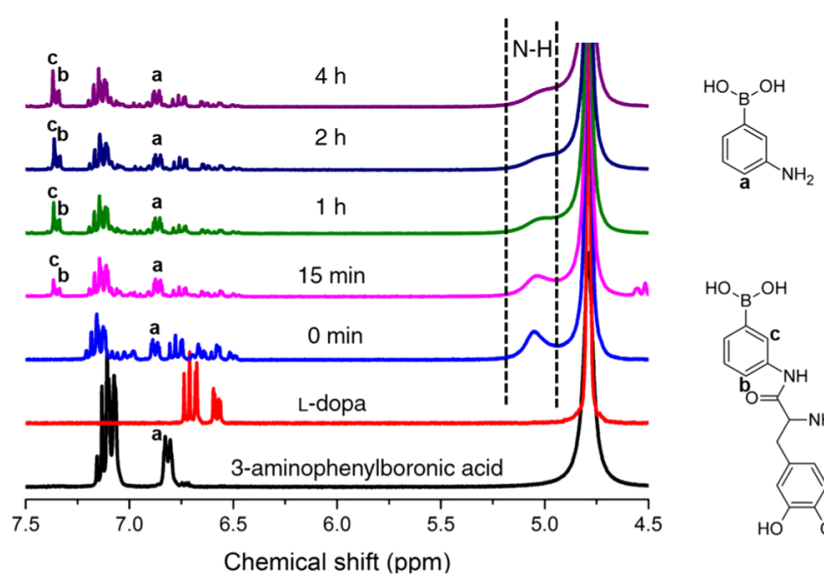

Figure 1. Time course of ${ }^{1} \mathrm{H}$ NMR spectra of the synthesized PBALD. Chemical shifts of the protons at the indicated positions are (a) 6.98-6.92 (m, 1H); (b) $7.42(\mathrm{~d}, J=1.7 \mathrm{~Hz}, 1 \mathrm{H})$; and (c) $7.45(\mathrm{~s}$, $1 \mathrm{H})$.

product yield of $40 \%$. Thus, the PBA was successfully introduced to the LD monomer. Because the $\mathrm{p} K_{\mathrm{a}}$ value of 3amino PBA is higher than that of other PBA derivatives in the bulk aqueous solution $\left(\mathrm{p} K_{\mathrm{a}}: 8.9\right)$, we consider that the PBALD complex by di-ester binding has not been formed during the synthesis process at neutral $\mathrm{pH}^{25}$ Due to the difficulty in the purification of $\mathrm{PBA}-\mathrm{LD}$, the reaction mixture was directly used for the oxidative copolymerization of PBA-LD and LD to form the polymer coating. The oxidation of CA is proposed to be a multistep process requiring an external source of electrons. Thus, the formation of poly $(\mathrm{PBA}-\mathrm{LD} / \mathrm{LD})$ was monitored through the current changes of the $\mathrm{Au}$ electrode. Figure 2 shows the time course of current changes in the LD

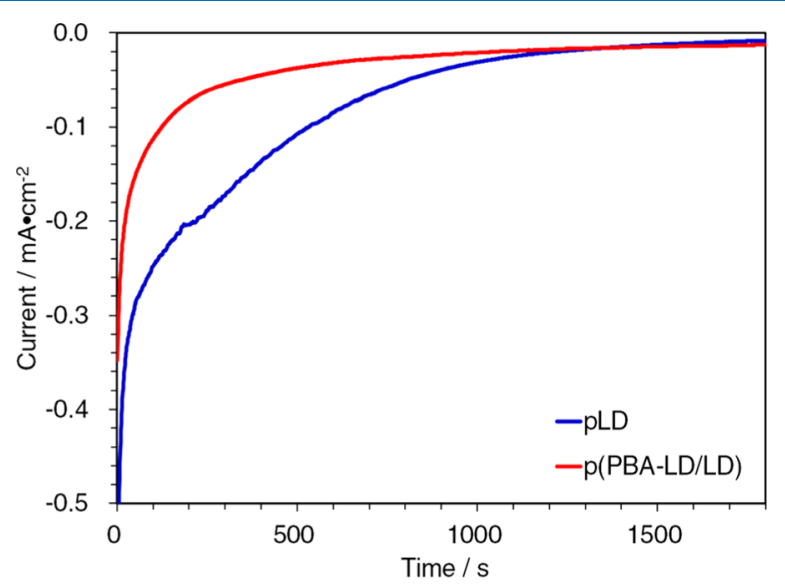

Figure 2. Time course of current change in the electropolymerization synthesis of poly $(\mathrm{LD})$ and poly $(\mathrm{PBA}-\mathrm{LD} / \mathrm{LD})$ on an Au surface at a constant voltage $(0.5 \mathrm{~V})$.

and $\mathrm{PBA}-\mathrm{LD} / \mathrm{LD}$-containing solutions at a constant positive potential. A negative current flowed to the $\mathrm{Au}$ electrode because $\mathrm{Au}$ accepted electrons from the solutions. Furthermore, the current increase almost stopped after $1500 \mathrm{~s}$, suggesting that the Au electrode was sufficiently covered with an insulating polymer film. From these results, it was suggested that poly(LD) and poly(PBA-LD/LD) layers had accumulated on the $\mathrm{Au}$ surface. After the electropolymerization of poly $(\mathrm{LD})$ and poly $(\mathrm{PBA}-\mathrm{LD} / \mathrm{LD})$, the thickness of the polymer layers was evaluated by ellipsometric analysis (Figure S1, Supporting Information). The thickness of poly(LD) was $13.9 \pm 0.1 \mathrm{~nm}$, whereas that of poly $(\mathrm{PBA}-\mathrm{LD} / \mathrm{LD})$ was $3.5 \pm$ $0.1 \mathrm{~nm}$. It is well known that poly(LD) accumulates and strongly adheres to the metal surfaces, such as $\mathrm{Au}$, in nanometer thickness and that the polymer chains orient to form multilayers through $\pi-\pi$ stacking and electrostatic interactions. $^{26,27}$ The lamination mechanism was proposed to start with a few nanometer-thick polycatecholamine layers accumulating by $\pi-\pi$ stacking as a fundamental nanoaggregate, followed by secondary nanoaggregation to $10-20 \mathrm{~nm}$ thick substructures through $\pi-\pi$ stacking of the edge-to-edge nanoaggregates. ${ }^{28,29}$ From this information, it was supposed that the poly $(\mathrm{PBA}-\mathrm{LD} / \mathrm{LD})$ layer formed the fundamental nanoaggregates, whereas the poly $(\mathrm{LD})$ layer was grown as the secondary aggregate. In the case of poly $(\mathrm{PBA}-\mathrm{LD} / \mathrm{LD})$, the PBA side chain in the nanoaggregates of poly $(\mathrm{PBA}-\mathrm{LD} / \mathrm{LD})$ inhibited the edge-to-edge $\pi-\pi$ stacking of poly(LD) and suppressed the thicker layer growth of the secondary aggregate substructure. The wettability of each surface also supported the successful fabrication of a PBA-containing poly(LD) layer. Figure 3 shows the water static contact angles of $\mathrm{Au}$,

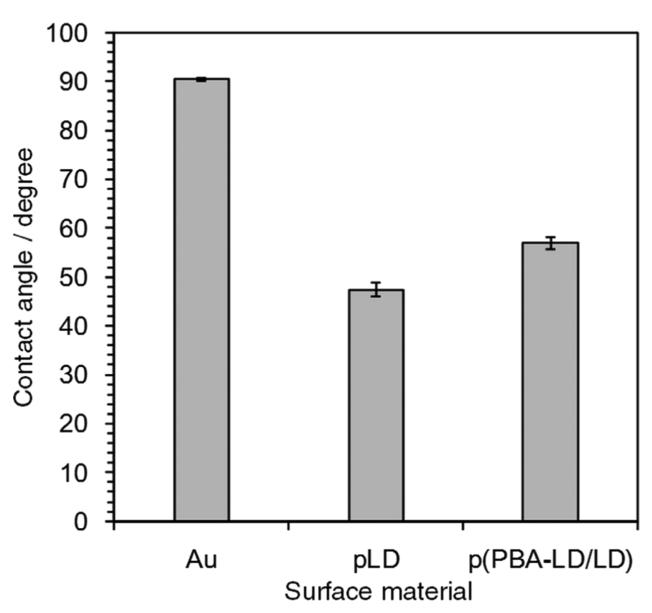

Figure 3. Static water contact angles of each layer-modified $\mathrm{Au}$ surface.

poly(LD)-coated $\mathrm{Au}$, and poly(PBA-LD/LD)-coated Au. Lamination with poly $(\mathrm{LD})$ produced a more hydrophilic $\mathrm{Au}$ surface, consistent with previous reports. ${ }^{12}$ The contact angle of the poly(PBA-LD/LD)-coated Au was slightly larger than that of poly $(\mathrm{LD})$, possibly because the surface of poly(PBA$\mathrm{LD} / \mathrm{LD}$ ) contained hydrophobic benzene rings from the PBA unit. Note that a hydrophilic carboxyl group is present at the corresponding position of $\mathrm{LD}$.

Detection of CA Using the Poly(PBA-LD/LD)-Coated Gate FET on an Au Surface. To investigate the electrochemical CA sensor, we assembled a poly(PBA-LD/LD)coated extended-gate FET by connecting the nanolayered poly(PBA-LD/LD)-coated Au electrode with a MOSFET. First, the interfacial property change by introducing the PBA unit to the poly(LD) layer was examined using a semiconductor parameter analyzer. Changes in the drain current $\left(I_{\mathrm{D}}\right)$ were measured at the time of applying the gate voltage, from -2 to $+3 \mathrm{~V}$, and the threshold of the gate voltage shift $\left(\Delta V_{\mathrm{T}}\right)$ at $4 \mathrm{~mA}$ of $I_{\mathrm{D}}$ was plotted against changes in $\mathrm{pH}$ (from 5 to 11 ) with the addition of $10 \mu \mathrm{M}$ catechol addition in 100 $\mathrm{mM}$ sodium phosphate buffer. Figure 4 shows the $\Delta V_{\mathrm{T}}$ plots of 
(A)

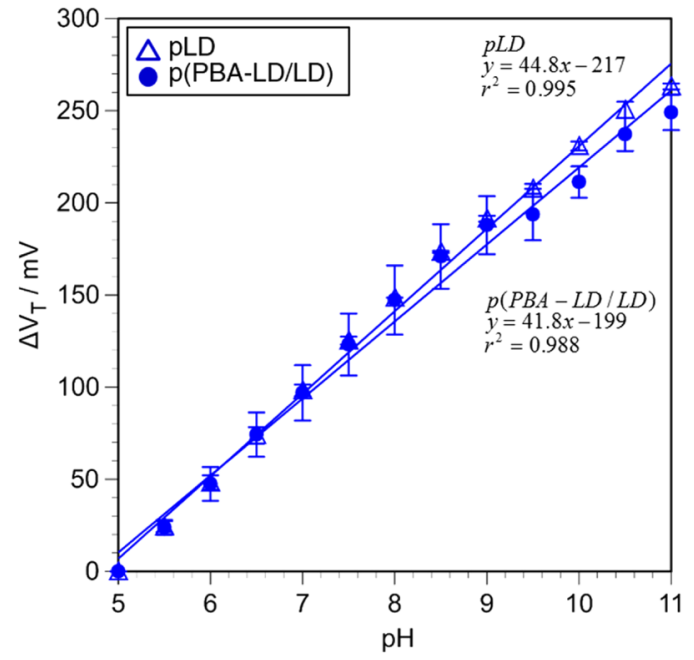

(B)

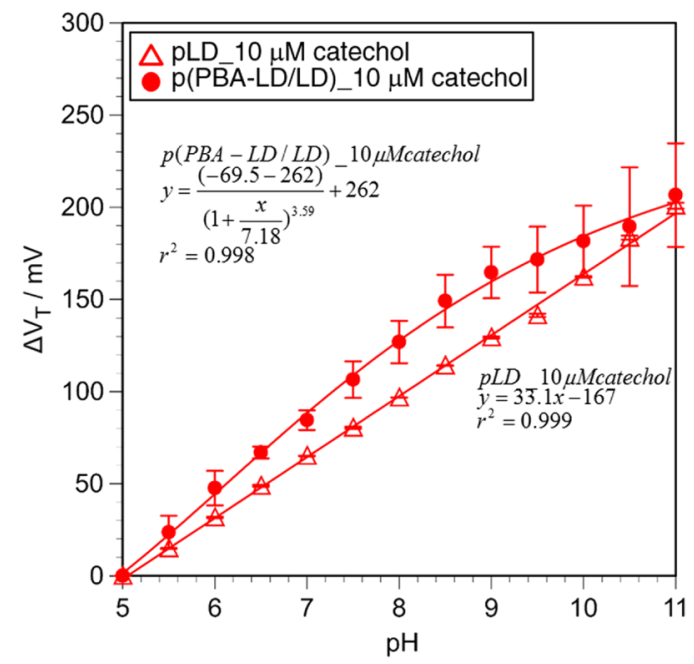

Figure 4. Threshold gate potential shift $\left(\Delta V_{\mathrm{T}}\right)$ in poly $(\mathrm{LD})$ - and poly $(\mathrm{PBA}-\mathrm{LD} / \mathrm{LD})$-coated gate FET at $4 \mathrm{~mA}$ of $I_{\mathrm{D}}$ from the $I_{\mathrm{D}}-V_{\mathrm{G}}$ curve as the $\mathrm{pH}$ changes from 5 to 11 in $100 \mathrm{mM}$ of sodium phosphate buffer (A) without and (B) with $10 \mu \mathrm{M}$ catechol. Linear fitted lines were obtained for poly $(\mathrm{LD})$; sigmoidal curve was obtained for the poly(PBA-LD/LD)-coated gate FET with $10 \mu \mathrm{M}$ of $\mathrm{CA}$ in each pH solution, as indicated with red dots.

the poly $(\mathrm{LD})$ - and poly $(\mathrm{PBA}-\mathrm{LD} / \mathrm{LD})$-coated gate FETs from the obtained $I_{\mathrm{D}}-V_{\mathrm{G}}$ curve (Figure $\mathrm{S} 2$ in the Supporting Information). In the catechol-free case (Figure 4A), both FETs obviously showed a linear $\mathrm{pH}$ response. This was caused by the functional side chains in LD, such as the carboxyl and amino groups, equilibrating with protons. ${ }^{30}$ The $\Delta V_{\mathrm{T}}$ shift was almost the same in both the poly(LD)- and poly(PBA-LD/LD)coated gate FETs (ca. $45 \mathrm{mV} / \mathrm{pH}$ for poly(LD) and $42 \mathrm{mV}$ / $\mathrm{pH}$ for poly $(\mathrm{PBA}-\mathrm{LD} / \mathrm{LD}))$. On the contrary, the $\Delta V_{\mathrm{T}}$ shift in the poly $(\mathrm{LD})$-coated gate $\mathrm{FET}$ decreased to $33 \mathrm{mV} / \mathrm{pH}$, and the plots in the poly $(\mathrm{PBA}-\mathrm{LD} / \mathrm{LD})$-coated gate FET changed to a sigmoidal curve when $10 \mu \mathrm{M}$ of catechol was added (Figure 4B). Considering the decreasing $\mathrm{pH}$ sensitivity of poly(LD)-coated gate FET from $44.8 \mathrm{mV} / \mathrm{pH}$ in the buffer without catechol to $33.1 \mathrm{mV} / \mathrm{pH}$ in that with catechol, catechol most likely attached to the poly(LD) surface through $\pi-\pi$ stacking and hydrogen bonding. Thus, the attached catechol shielded the functional groups of poly(LD), preventing the participation of the proton equilibrium. Thus, curve fitting of the plots of the poly(PBA-LD/LD)-coated gate $\mathrm{FET}$ in the presence of $10 \mu \mathrm{M}$ catechol was changed from the linear to sigmoidal. The $\Delta V_{\mathrm{T}}$ shift was sharp near the neutral $\mathrm{pH}$ region and weakened as the $\mathrm{pH}$ exceeded 9, resulting in an upward fitting curve. This result indicated that the PBA equilibrium with catechol affected the change in the $\Delta V_{\mathrm{T}}$ shift. The decrease in $\Delta V_{\mathrm{T}}$ at a higher $\mathrm{pH}$ was possibly caused by the negatively charged boron atom forming a complex with PBA and catechol. This might prove the existence of PBA units on the surface of poly (PBA-LD/LD) layer, and the $\mathrm{p} K_{\mathrm{a}}$ of PBA at the interface could be calculated to be 7.18 by fitting the sigmoidal curve (eq 1$).^{31}$

$$
\Delta V_{\mathrm{T}}=\frac{\left(V_{\mathrm{T}_{\min }}-V_{\mathrm{T}_{\max }}\right)}{\left[1+\left(\frac{\mathrm{pH}}{\mathrm{pK}}\right)^{n}\right]}+V_{\mathrm{T}_{\max }}
$$

where $\Delta V_{\mathrm{T}_{\min }}$ and $\Delta V_{\mathrm{T}_{\max }}$ are approximated values of the minimum and maximum threshold gate voltage, respectively, $\mathrm{p} K_{\mathrm{a}}$ is the acid dissociation constant of the poly (PBA-LD/
LD) layer, and $n$ is the Hill coefficient (slope at the $\mathrm{p} K_{\mathrm{a}}$ point). PBA maintains an equilibrium between four states in the presence of diol compounds, as shown in Scheme 1B. The acidic dissociation constant $\left(\mathrm{p} K_{\mathrm{a} \text {-acid }}\right)$ of PBA is reported to be 8.6-8.8, in which the boron atom orbital changes from $\mathrm{sp}^{2}$ to $\mathrm{sp}^{3}$ with increasing negative charge (state 2 in Scheme 1B). In this experiment, we calculated the $\mathrm{p} K_{\mathrm{a}}$ of $\mathrm{PBA}$ in the tetrahedral binding state with catechol (state 3 in Scheme 1B). Actually, the $\mathrm{p} K_{\mathrm{a}}$ of the PBA situated at the interface between the electrode and solution was approximately 7 , indicating that the surface potential changes derived from PBA equilibrium with catechol could be monitored at the physiological $\mathrm{pH}$.

From the kinetic analysis of the poly (PBA-LD/LD)-coated gate FET interface, each type of CA detection was examined by changes in the surface potential $\left(V_{\text {out }}\right)$ of the FET using a realtime source-follower monitoring system. Each CA was gradually added, from $1 \mathrm{nM}$ to $20 \mu \mathrm{M}$, final concentration in PBS buffer $(\mathrm{pH}=7.4)$ onto the poly $(\mathrm{PBA}-\mathrm{LD} / \mathrm{LD})$-coated $\mathrm{Au}$, and the $V_{\text {out }}$ changes were monitored as shown in Figure 5. The $V_{\text {out }}$ decreased in the negative direction whenever CA was added, indicating that the negatively charged PBA units were formed by the binding with CAs and recognized by the FET. On the other hand, the $V_{\text {out }}$ of the poly(LD)-coated gate FET hardly responded to the CAs inputs, except for the higher LD concentration case (Figure S3, the Supporting Information). The interaction between PBA and CAs was also confirmed by attenuated total reflection-Fourier transform infrared (ATRFTIR) spectroscopic analysis of poly(LD)- and poly(PBA$\mathrm{LD} / \mathrm{LD}$ )-coated $\mathrm{Au}$ electrodes. The peaks of $\mathrm{C}-\mathrm{O}$ stretching at $1256 \mathrm{~cm}^{-1}, \mathrm{~N}-\mathrm{H}$ stretching at $1309 \mathrm{~cm}^{-1}$, and $\mathrm{C}=\mathrm{O}$ stretching at $1739 \mathrm{~cm}^{-1}$ increased and shifted, and specific peaks derived from di-ester binding of boronic acid and free CAs appeared after addition of CAs in poly(PBA-LD/LD)coated $\mathrm{Au}$, compared with those in poly(LD)-coated $\mathrm{Au}$ (Figure S4, the Supporting Information). Considering the relationship between the gate potential shift in the FET and the number of PBA units reacting with CAs, the $V_{\text {out }}$ of the poly(PBA-LD/LD)-coated gate FET shifted $80-180 \mathrm{mV}$ with 


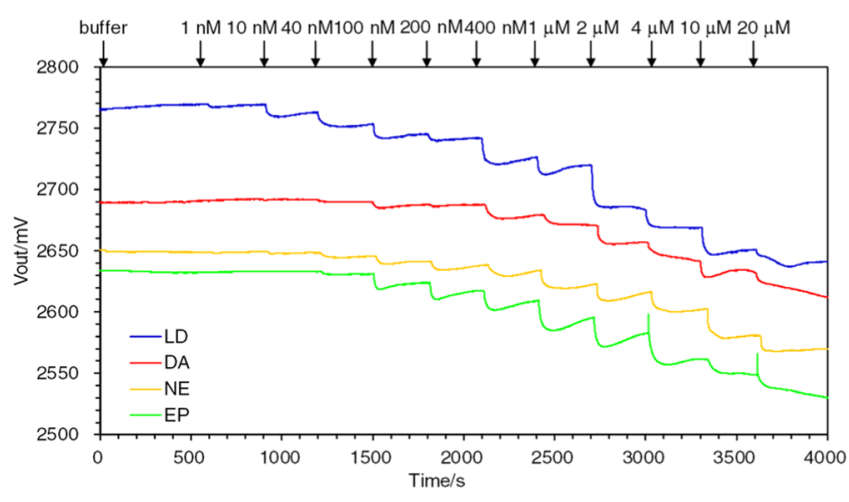

Figure 5. Time course for $V_{\text {out }}$ of poly $(\mathrm{PBA}-\mathrm{LD} / \mathrm{LD})$-coated gate FET at different concentrations of CAs at a constant source-drain current $(100 \mu \mathrm{A})$. Arrows indicate time and final concentration of CA addition. $V_{\text {out }}$ of poly(PBA-LD/LD)-coated gate FET upon LD addition (blue line), DA addition (red line), NE addition (orange line), and EP addition (green line).

every $10 \mu \mathrm{M}$ addition of each type of CA. The capacitance model at the device interface can be described using eq 2

$$
\Delta \mathrm{Q}_{\mathrm{PBA}-\mathrm{CA}}=C_{\text {total }} \cdot \Delta V_{\text {out }}
$$

where $\Delta Q_{\mathrm{PBA}-\mathrm{CA}}$ is the charge change from the equilibrium of PBA and CA and $C_{\text {total }}$ is the combined capacitance of the entire circuit. Figure 6 shows a circuit diagram of the combined

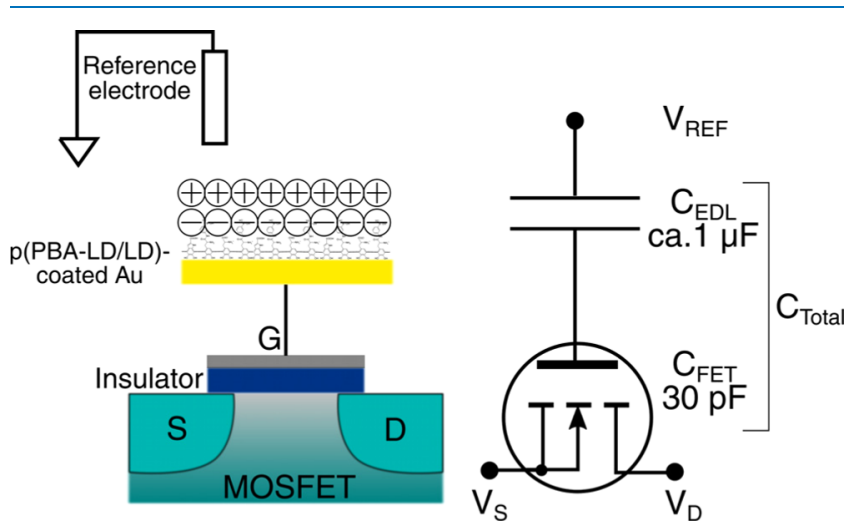

Figure 6. Circuit diagram of the combined capacitance consisting of the electric double-layer capacitance at poly $(\mathrm{PBA}-\mathrm{LD} / \mathrm{LD})$-coated gate electrode interface and the MOSFET capacitance.

capacitance model. $C_{\text {total }}$ may be the capacitance model in series from the capacitance of the MOSFET device itself and the electric double-layer capacitance at the poly (PBA-LD/ $\mathrm{LD})$-coated gate electrode interface. However, $C_{\text {total }}$ is assumed to dominate the MOSFET value because the MOSFET capacitance $\left(C_{\mathrm{FET}}=30 \mathrm{pF}\right)$ was quite small compared to the electric double-layer capacitance at the poly(PBA-LD/LD)coated gate electrode interface $\left(C_{\mathrm{EDL}}=\sim 1 \mu \mathrm{F}\right)$. Thus, there was a significant change of $3.0 \times 10^{-12} \mathrm{C}$ when the $V_{\text {out }}$ of the poly(PBA-LD/LD)-coated gate FET shifted $100 \mathrm{mV}$. Thus, the amount of diol binding to PBA was estimated to be $2.4 \times$ $10^{7}$ molecules $\mathrm{cm}^{-2}$. It is known that the range of the electric double layer decreases as the salt concentration increases presented by the Debye length. In the present study, poly (PBA-LD/LD) thin film exceeds the theoretical Debye length from the $\mathrm{Au}$ surface. However, because the charge change in the boronic acid molecule is obviously captured by the MOSFET, we have to reconsider the position of the diffusion layer in case of thin layer-coated $\mathrm{Au}$ surface, referring to the site-binding model at the oxide membrane.

Additionally, a difference in the gate potential changes was observed in the time course for sensitivity at lower concentration and gate potential shift magnitude between the different CAs (Figure 5). In particular, the poly (PBA-LD/ LD)-coated gate FET showed a higher sensitivity to LD because the $V_{\text {out }}$ decreases upon LD addition starting at $1 \mathrm{nM}$, whereas the other CAs barely caused the $V_{\text {out }}$ to decrease at 40 $n M$. To evaluate the affinity of PBA in the poly (PBA-LD/ LD)-coated gate FET with each $C A$, the kinetic parameters were calculated by fitting the $\Delta V_{\text {out }}$ at each concentration of $\mathrm{CA}$ to a Langmuir adsorption isotherm (eq 3 ).

$$
\Delta V_{\text {out }}=\frac{V_{\max }[\mathrm{CA}]}{1 / K_{\mathrm{s}}+[\mathrm{CA}]}
$$

where $V_{\max }$ is the maximum $V_{\text {out }}$ shift, reflecting the amount of each CA adsorption to the surface of poly(PBA-LD/LD), and $K_{\mathrm{s}}$ is the binding constant. Figure 7 shows the plots of $\Delta V_{\text {out }}$ at

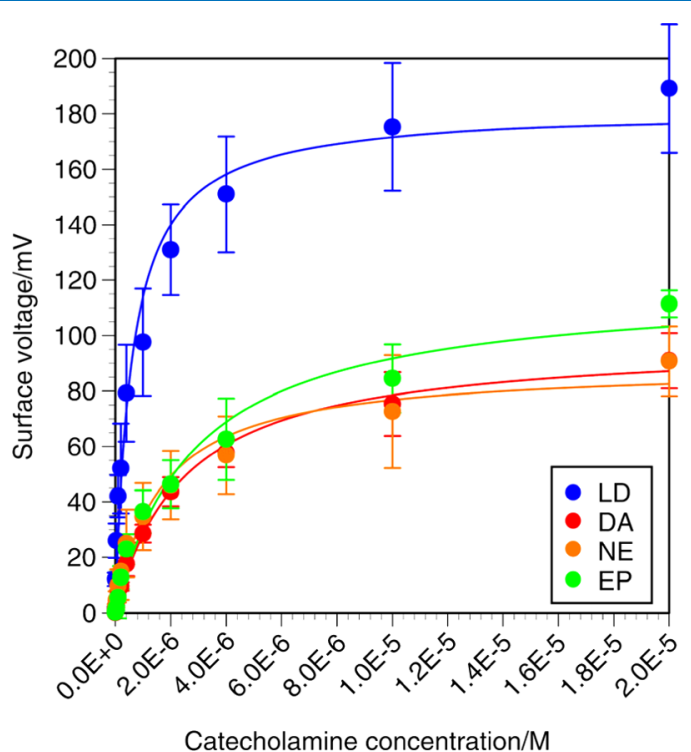

Figure 7. Plots of $\Delta V_{\text {out }}$ and the approximated curves using the Langmuir adsorption isotherm at different concentrations of CA addition from the real-time data of Figure 5. $\Delta V_{\text {out }}$ and fitted lines of poly (PBA-LD/LD)-coated gate FET with addition of LD (blue), DA (red), NE (orange), and EP (green).

each concentration of added CA from the real-time monitoring data in Figure 5 and curve fitting with the Langmuir adsorption isotherm. The kinetic parameters are summarized in Table 1.

Table 1. Kinetic Parameters Based on a Langmuir Adsorption Isotherm from the Gate Surface Potential Response of CA Addition to the Poly(PBA-LD/LD)Coated Gate FET in Figure 7

\begin{tabular}{ccccc} 
& \multicolumn{4}{c}{ kinetic parameters } \\
\cline { 2 - 5 } CA added & $\Delta V_{\max }(\mathrm{mV})$ & $K_{\mathrm{s}}\left(\times 10^{6} \mathrm{M}^{-1}\right)$ & $R^{2}$ & $u^{a}\left(\times 10^{6} \mathrm{M}^{-1}\right)$ \\
LD & 182 & 1.68 & 0.977 & 0.57 \\
$\mathrm{DA}$ & 98 & 0.40 & 0.995 & 0.04 \\
$\mathrm{NE}$ & 89 & 0.62 & 0.976 & 0.39 \\
EP & 118 & 0.35 & 0.980 & 0.14
\end{tabular}

${ }^{a}$ Standard measurement uncertainties of $K_{\mathrm{s}}$ value. 
In Figure 7, a clear difference was observed in the affinity of CAs to poly $(\mathrm{PBA}-\mathrm{LD} / \mathrm{LD})$. The kinetic parameters of $\mathrm{LD}$ were approximately 2 times higher than those of other CAs in $V_{\max }$ which reflected the amount of CA required for saturated adsorption to poly(PBA-LD/LD), and about 3-5 times higher in $K_{\mathrm{s}}$. Note that the poly(LD)-coated gate FET did not show a well-defined selectivity to $\mathrm{LD}$, indicating a significant role of PBA moieties in this device (Figure S3, the Supporting Information).

Concerning the association constants of CAs, the fluorescence and cyclic voltammetric analyses have been reported using chemically modified PBA molecules. Maue and Schrader developed a colorimetric sensor for CAs and evaluated the association constants of CAs to be $2.7 \times 10^{2} \mathrm{M}^{-1}$ in $\mathrm{LD}, 2.8 \times 10^{2} \mathrm{M}^{-1}$ in $\mathrm{DA}, 3.5 \times 10^{2} \mathrm{M}^{-1}$ in $\mathrm{NE}$, and $3.1 \times$ $10^{2} \mathrm{M}^{-1}$ in EP. ${ }^{32}$ Secor and Glass calculated the association constants of DA, NE, and EP to be $3.4 \times 10^{3}, 6.5 \times 10^{3}$, and $5.0 \times 10^{3} \mathrm{M}^{-1}$, respectively, to boronic acid-containing coumarin aldehyde by monitoring the fluorescence spectra. ${ }^{33}$ Additionally, the electrochemical sensing selectivity for catechol was studied using $N$-hexadecyl-pyridinium-4-boronic acid monolayer films, and the association constants of CAs were calculated to be $1.3 \times 10^{4} \mathrm{M}^{-1}$ in $\mathrm{LD}$ and $1.0 \times 10^{4} \mathrm{M}^{-1}$ in DA. ${ }^{33,34}$ In this study, 1 or 2 orders of magnitude higher $\mathrm{p} K_{\mathrm{a}}$ values were obtained from the poly $(\mathrm{PBA}-\mathrm{LD} / \mathrm{LD})$-coated gate $\mathrm{FET}$ than in the previous reports, indicating that the poly(PBA-LD/LD)-coated gate FET could detect CAs with higher sensitivity. In addition, the affinity to $\mathrm{LD}$ was improved in the poly(PBA-LD/LD)-coated electrode from both association constant and saturated adsorption amount aspects. This might be caused by the difference in the CA interactions with the poly $(\mathrm{LD})$-base layer. It is expected that CAs are attracted to the poly (PBA-LD/LD) surface by three binding forces: the di-ester binding to PBA, $\pi-\pi$ stacking of the benzene ring, and electrostatic interactions with the functional groups in the CA moiety. A computational study of PBA-based selective CA recognition and experimental research of selective fluorescence $\mathrm{LD}$ sensing utilized the three recognition points to explain the strong binding. ${ }^{35,36}$ In this study, because the affinity to PBA and $\pi-\pi$ stacking of the benzene ring can be considered to be similar to the previous reports, the amino group of the poly $(\mathrm{PBA}-\mathrm{LD} / \mathrm{LD})$ layer is the major difference. Thus, we propose that the amino group selectively attracts LD, which possesses a carboxyl group more acidic than the other CAs. We conclude that the carboxyl group of the LD side chain is the critical factor for the prominent affinity to the poly(PBA-LD/LD) surface.

The detection sensitivity of CAs was evaluated from the $\Delta V_{\text {out }}$ in the poly (PBA-LD/LD)-coated gate FET from 10 $\mathrm{nM}$ to $20 \mu \mathrm{M}$ for each concentration in Figure $5 .^{37}$ Semilogarithmic plots and their linear approximations between $\Delta V_{\text {out }}$ and CA concentration were obtained with a correlation coefficient in the range of 0.890-0.971 (Figure S5, the Supporting Information). The slope of the plots was $58.7 \mathrm{mV} /$ [LD] dec, $28.5 \mathrm{mV} /[\mathrm{DA}] \mathrm{dec}, 27.4 \mathrm{mV} /[\mathrm{NE}] \mathrm{dec}$, and 33.5 $\mathrm{mV} /[\mathrm{EP}]$ dec. The poly $(\mathrm{PBA}-\mathrm{LD} / \mathrm{LD})$-coated gate FET had a wide dynamic range from $10 \mathrm{nM}$ to $20 \mu \mathrm{M}$, and the limits of detection (LOD) were $3.5 \mathrm{nM}$ for $\mathrm{LD}, 12.0 \mathrm{nM}$ for DA, 7.5 $\mathrm{nM}$ for NE, and $12.6 \mathrm{nM}$ for EP. In Table S1, we compare our results on $\mathrm{CA}$ and catechol detection with those from other works that used electrochemical and fluorescent methods. The poly (PBA-LD/LD)-coated gate FET is superior to the other sensors using various modified interfaces because it exhibits a lower limit of detection (LOD) and a wider linear range. It is considered that the sensitivity to $\mathrm{CA}$ is achieved by a combination of a well-controlled, nanolayer interface of PBA molecules containing poly(LD) and the FET that can detect charge changes at the interface directly. Moreover, the poly $(\mathrm{PBA}-\mathrm{LD} / \mathrm{LD})$ is easily fabricated on the electrode, making it suitable for practical applications in diagnostic sensors. In blood plasma, the normal LD concentration is ca. 30-40 nM, whereas the other CAs exist in much lower concentrations. $^{38,39}$ In urine, the normal CA concentration is reported as follows: below $10 \mu \mathrm{M}$ for $\mathrm{LD}, 600 \mathrm{nM}$ to $5 \mu \mathrm{M}$ for $\mathrm{DA}$, and $100-800 \mathrm{nM}$ for $\mathrm{NE}$ and EP combined. ${ }^{40,41}$ In lacrimal fluid, each concentration of CA in healthy individuals is reported to be $2 \mu \mathrm{M}$ for $\mathrm{LD}, 30 \mu \mathrm{M}$ for $\mathrm{DA}$, and $1.5 \mu \mathrm{M}$ for NE and EP combined. ${ }^{42}$ From these reports, it is apparent that the poly(PBA-LD/LD)-coated gate FET has sufficient sensitivity for the $\mathrm{CA}$ detection in various bodily fluid. In particular, the highly sensitive detection of LD is expected to play a role in the early detection and diagnosis of Parkinson's disease. Furthermore, the monitoring of LD concentrations is required because administrated $\mathrm{LD}$, as a therapeutic agent for Parkinson's disease, has a risk of causing serious side effects.

CA sensors may have several applications in in vivo and in vitro diagnostic devices because the poly(PBA-LD/LD)coated gate FET is easy to fabricate and reduces noise from the biological fluid components. Furthermore, its self-polymerization property makes it biocompatible with maintaining superior CA sensitivity. Room still exists for increasing the specificity and accuracy of this FET before practical applications are considered; thus, further investigations are required along with in vivo studies.

\section{CONCLUSIONS}

In this study, we investigated the fundamental properties of the poly (PBA-LD/LD)-coated gate FET. PBA was introduced to $\mathrm{LD}$ and electropolymerized on the surface of an $\mathrm{Au}$ gate electrode. The poly(PBA-LD/LD)-coated Au electrode was connected to a MOSFET as an extended-gate electrode and the electrochemical detection of CAs was carried out using FET measurements. The gate surface potential in the poly(PBA-LD/LD)-coated gate FET clearly decreased after $1 \mathrm{nM}$ of LD addition or $40 \mathrm{nM}$ addition of other CAs. The affinity of CAs to the poly(PBA-LD/LD)-coated gate FET was estimated from the gate potential shift using the Langmuir adsorption isotherm. Obvious differences in the saturated binding amount $\left(V_{\max }\right)$ and binding constant $\left(K_{\mathrm{s}}\right)$ were found between $\mathrm{LD}$ and other CAs investigated. The poly $(\mathrm{PBA}-\mathrm{LD} /$ LD)-coated gate FET may be useful for highly sensitive and accurate biosensing of CAs in various bodily fluids.

\section{EXPERIMENTAL SECTION}

Chemicals and Reagents. All the chemicals were obtained from commercial suppliers and used without further purification. 3-(3,4-Dihydroxyphenyl)-L-alanine (L-dopa, LD), 3-hydroxytyramine hydrochloride (dopamine, DA), L-norepinephrine bitartrate (NE), and L-epinephrine (EP) were purchased from Tokyo Kasei Industry (Japan). For the synthesis of the phenylboronic acid-LD derivative, 3-aminophenylboronic acid monohydrate $\left(\mathrm{NH}_{2}\right.$-PBA, Sigma-Aldrich) and 4-(4,6-dimethoxy-1,3,5-triazin-2-yl)-4-methylmorpholinium chloride (DMT-MM, Wako Pure Chemical, Japan) were used. The n-channel metal-oxide semiconductor FET 
(MOSFET, 2N7002) was purchased from NXP Semiconductors. Ultrapure water (Ul-pure, $18.2 \mathrm{M} \Omega \mathrm{cm}$ of purity, Komatsu Electronics Co., Ltd.) was used in all the experiments.

Chemical Synthesis of PBA-LD Derivative and Electropolymerized Formation of Poly(PBA-LD/LD) on an Au Electrode. The LD PBA-functionalized monomer was synthesized by mixing $\mathrm{LD}(10 \mathrm{mM})$ and $\mathrm{NH}_{2}$-PBA $(10 \mathrm{mM})$ with DMT-MM $(10 \mathrm{mM})$, as a dehydration-condensation reagent, in $10 \mathrm{~mL}$ of mixed solvents (water/methanol, $1: 1 \mathrm{v} /$ $\mathrm{v}$ ). Dehydration-condensation proceeded for $4 \mathrm{~h}$ at room temperature by inverting the sealed reaction tube. The resulting mixture was washed with water and the organic layer was concentrated using a rotary evaporator. The obtained solid was dried in vacuo $(0.3 \mathrm{~g}, 90 \%$ yield $)$. The structure of the synthesized product was confirmed by ${ }^{1} \mathrm{H}$ NMR. The coordinate peak allowed for us to identify the resulting product: ${ }^{1} \mathrm{H}$ NMR (300 MHz, $\left.\mathrm{CD}_{3} \mathrm{OD}\right): \delta 7.45$ (s, 1H), 7.42 $(\mathrm{d}, J=1.7 \mathrm{~Hz}, 1 \mathrm{H}), 6.98-6.92(\mathrm{~m}, 1 \mathrm{H})$. The synthesized PBA-LD and pure LD were copolymerized auto-oxidatively. Tris(hydroxymethyl)aminomethane $(100 \mathrm{mM})$ was dissolved in an aqueous solution of PBA-LD. Thereafter, $500 \mu \mathrm{L}$ aliquot of the PBA-LD-containing solution was directly dispensed to a $10 \mathrm{~mm}$ diameter glass ring attached to the Au electrode. After connecting the $\mathrm{Au}$ electrode as a working electrode, $\mathrm{Ag} / \mathrm{AgCl}$ electrode as a reference, and platinum as a counter electrode, oxidative polymerization was carried out by applying $0.5 \mathrm{~V}$ to the working electrode for $30 \mathrm{~min}$ using an electrochemical analyzer (ALS614E, BAS Inc., Japan).

Surface Analysis of the Polymerized Poly(PBA-LD/ LD) Layer-Coated Au Electrode. The thicknesses of poly (LD) and poly (PBA-LD/LD) on the Au electrodes were estimated by the ellipsometric analysis using a rotating ellipsometer (model M2000U, J.A. Woollam Co., Inc.) and WVASE32 software. The wavelength of the incident light ranged between 200 and $1600 \mathrm{~nm}$, and the incident light angled from 65 to $80^{\circ}$ in $5^{\circ}$ steps. To calculate the thickness of each layer, the experimental spectra were fitted using the Cauchy model. The thickness was determined as the average of at least three distinct measurements from different locations on the substrate. The formation of poly $(\mathrm{LD})$ and poly $(\mathrm{PBA}-\mathrm{LD} /$ $\mathrm{LD})$ was checked by measuring the static water contact angle using a CA-W automatic contact-angle meter (Kyowa Interface Science, Japan). Two microliters of Ul-pure water was placed on the surface of each substrate under ambient conditions. The contact angles were monitored using a charge-coupled device camera, and the captured images were analyzed using FAMAS software (Kyowa Interface Science). The contact angles were calculated as the average of ten measurements taken at different positions. The ATR-FTIR measurements were performed using a FT/IR 4200 (JASCO, Japan). ZnSe was used as the crystal plate, and all the spectra were recorded over the wave number range from 4000 to $600 \mathrm{~cm}^{-1}$.

Electrochemical Measurements of the Poly(PBA-LD/ LD)-Coated Gate FET. To clarify the interfacial properties of the polymer-modified surface, poly(LD) and poly (PBA-LD/ $\mathrm{LD}$ )-coated $\mathrm{Au}$ electrode was connected to the gate of an nchannel MOSFET device using crocodile clip, and the threshold gate voltage shift $\left(\Delta V_{\mathrm{T}}\right)$ at a drain current $\left(I_{\mathrm{D}}\right)$ of $4 \mathrm{~mA}$ and drain voltage $\left(V_{\mathrm{D}}\right)$ of $2 \mathrm{~V}$ was measured using a semiconductor parameter analyzer (B1500A, Keysight technologies). Sodium phosphate buffer $(100 \mathrm{mM})$ from $\mathrm{pH} 5$ to 12 with/without $10 \mu \mathrm{M}$ of catechol was used as the $\mathrm{pH}$ solution. The $\mathrm{p} K_{\mathrm{a}}$ was calculated by fitting the $\Delta V_{\mathrm{T}}$ versus $\mathrm{pH}$ data to a sigmoidal curve. To evaluate the affinity of the poly $(\mathrm{PBA}-\mathrm{LD} / \mathrm{LD})$-coated gate $\mathrm{FET}$, the output voltage response to each $\mathrm{CA}$ was measured using a real-time monitoring system. Five hundred microliters of phosphate buffered saline (PBS, $\mathrm{pH}=7.4$ ) was poured into the poly(PBA-LD/LD)-coated gate FET equipped with a 10 $\mathrm{mm}$ diameter glass ring and equilibrated until the output voltage was stabilized. Thereafter, each CA was titrated gradually from $1 \mathrm{nM}$ to $20 \mu \mathrm{M}$. To suppress the gate surface potential spike signal, owing to the effect of sample addition, the injection volume was $1 \%$ of the total measurement solution volume. The time course of the surface potential at the Au gate interface was observed using a real-time FET source-follower circuit monitoring system. Changes in the gate surface potential $\left(V_{\text {out }}\right)$ were monitored by adjusting the constant drain-source current $\left(I_{\mathrm{DS}}\right)$ at $100 \mu \mathrm{A}$ by connecting to an $\mathrm{Ag} /$ $\mathrm{AgCl}$ reference electrode whose potential was set as $2.5 \mathrm{~V}$ of the reference potential.

\section{ASSOCIATED CONTENT}

\section{Supporting Information}

The Supporting Information is available free of charge on the ACS Publications website at DOI: 10.1021/acsomega. 8 b00518.

Ellipsometric data of the poly(LD) and poly(PBA-LD/ $\mathrm{LD}$ ) layer on the Au surface (Figure $S 1), I_{\mathrm{D}}-V_{\mathrm{G}}$ curve of poly(LD)- and poly(PBA-LD/LD)-coated gate FET in different $\mathrm{pH}$ solutions (Figure S2), the time course for gate surface potential of the poly(LD)-coated gate FET upon addition of CAs (Figure S3), ATR-FTIR data of poly(LD)- and poly(PBA-LD/LD)-coated Au electrode at $\mathrm{CA}$ addition (Figure S4), and semi-logarithmic plots of the gate surface potential shift versus each CA concentration (Figure S5), comparison with other sensors for the determination of catecholamine and catechol (Table S1) (PDF)

\section{AUTHOR INFORMATION}

\section{Corresponding Author}

*E-mail: kajisa@provigate.com.

ORCID

Taira Kajisa: 0000-0001-6121-9274

Tsuyoshi Michinobu: 0000-0001-6948-1189

\section{Author Contributions}

The manuscript was written through contributions of all the authors. All the authors have given approval to the final version of the manuscript.

\section{Funding}

This study was partly supported by the New Energy and Industrial Technology Development Organization (NEDO) under the Ministry of Economy Trade and Industry (METI) of Japan.

\section{Notes}

The authors declare no competing financial interest.

\section{ACKNOWLEDGMENTS}

Part of this work was conducted at the Advanced Characterization Nanotechnology Platform of the University of Tokyo, supported by the "Nanotechnology Platform" of the Ministry of Education, Culture, Sports, Science and Technology 
(MEXT), Japan. The authors wish to thank Assistant Professor $\mathrm{K}$. Konishi of the University of Tokyo in Japan for his help in the ellipsometry experiment.

\section{ABBREVIATIONS}

MOSFET, metal-oxide semiconductor field-effect transistor; PBA, phenylboronic acid; CA, catecholamine; DA, dopamine; $\mathrm{LD}$, L-dopa; NE, norepinephrine; EP, epinephrine

\section{REFERENCES}

(1) Goldstein, D. S.; Mccarty, R.; Polinsky, R. J.; Kopin, I. J. Relationship between Plasma Norepinephrine and Sympathetic Neural Activity. Hypertension 1983, 5, 552-559.

(2) Schwaber, M. K.; Glasscock, M. E.; Jackson, C. G.; Nissen, A. J.; Smith, P. G. Diagnosis and Management of Catecholamine Secreting Glomus Tumors. Laryngoscope 1984, 94, 1008-1015.

(3) Cawood, T. J.; Hunt, P.; O'Shea, D.; Cole, D.; Soule, S. Recommended Evaluation of Adrenal Incidentalomas Is Costly, Has High False-Positive Rates and Confers a Risk of Fatal Cancer That Is Similar to the Risk of the Adrenal Lesion Becoming Malignant; Time for a Rethink? Eur. J. Endocrinol. 2009, 161, 513-527.

(4) Wade, L. A.; Katzman, R. Synthetic Amino Acids and the Nature of L-Dopa Transport at the Blood-Brain Barrier. J. Neurochem. 1975, 25, 837-842.

(5) Pardridge, W. M. Brain Metabolism: A Perspective from the Blood-Brain Barrier. Physiol. Rev. 1983, 63, 1481-1535.

(6) Chalmers, J. P.; Baldessarini, R.; Wurtman, R. Effects of L-Dopa on Norepinephrine Metabolism in the Brain. Proc. Natl. Acad. Sci. U.S.A. 1971, 68, 662-666.

(7) Fotopoulou, M. A.; Ioannou, P. C. Post-Column Terbium Complexation and Sensitized Fluorescence Detection for the Determination of Norepinephrine, Epinephrine and Dopamine Using High-Performance Liquid Chromatography. Anal. Chim. Acta 2002, 462, 179-185.

(8) Woo, H. I.; Yang, J. S.; Oh, H. J.; Cho, Y. Y.; Kim, J. H.; Park, H.-D.; Lee, S.-Y. A Simple and Rapid Analytical Method Based on Solid-Phase Extraction and Liquid Chromatography-Tandem Mass Spectrometry for the Simultaneous Determination of Free Catecholamines and Metanephrines in Urine and Its Application to Routine Clinical Analysis. Clin. Biochem. 2016, 49, 573-579.

(9) Lee, H.; Dellatore, S. M.; Miller, W. M.; Messersmith, P. B. Mussel-Inspired Surface Chemistry for Multifunctional Coatings. Science 2007, 318, 426-430.

(10) Coyne, K. J.; Qin, X. X.; Waite, J. H. Extensible Collagen in Mussel Byssus: A Natural Block Copolymer. Science 1997, 277, $1830-1832$.

(11) Fei, B.; Qian, B.; Yang, Z.; Wang, R.; Liu, W.; Mak, C.; Xin, J. H. Coating Carbon Nanotubes by Spontaneous Oxidative Polymerization of Dopamine. Carbon 2008, 46, 1795-1797.

(12) Zhu, L.-P.; Yu, J.-Z.; Xu, Y.-Y.; Xi, Z.-Y.; Zhu, B.-K. Surface Modification of Pvdf Porous Membranes Via Poly (Dopa) Coating and Heparin Immobilization. Colloids Surf., B 2009, 69, 152-155.

(13) Zhang, M.; He, X.; Chen, L.; Zhang, Y. Preparation of Ida-Cu Functionalized Core-Satellite $\mathrm{Fe}_{3} \mathrm{O}_{4}$ /Polydopamine/Au Magnetic Nanocomposites and Their Application for Depletion of Abundant Protein in Bovine Blood. J. Mater. Chem. 2010, 20, 10696-10704.

(14) Mrówczyński, R.; Turcu, R.; Leostean, C.; Scheidt, H. A.; Liebscher, J. New Versatile Polydopamine Coated Functionalized Magnetic Nanoparticles. Mater. Chem. Phys. 2013, 138, 295-302.

(15) Bergveld, P. Development of an Ion-Sensitive Solid-State Device for Neurophysiological Measurements. IEEE Trans. Biomed. Eng. 1970, 17, 70-71.

(16) Fromherz, P.; Offenhausser, A.; Vetter, T.; Weis, J. A NeuronSilicon Junction: A Retzius Cell of the Leech on an Insulated-Gate Field-Effect Transistor. Science 1991, 252, 1290-1293.

(17) Lahav, M.; Kharitonov, A. B.; Willner, I. Imprinting of Chiral Molecular Recognition Sites in Thin $\mathrm{TiO}_{2}$ Films Associated with
Field-Effect Transistors: Novel Functionalized Devices for Chiroselective and Chirospecific Analyses. Chem. Eur. J. 2001, 7, 3992-3997.

(18) Sakata, T.; Kamahori, M.; Miyahara, Y. DNA Analysis Chip Based on Field-Effect Transistors. Jpn. J. Appl. Phys. 2005, 44, 28542859.

(19) Star, A.; Tu, E.; Niemann, J.; Gabriel, J. C. P.; Joiner, C. S.; Valcke, C. Label-Free Detection of DNA Hybridization Using Carbon Nanotube Network Field-Effect Transistors. Proc. Natl. Acad. Sci. U.S.A. 2006, 103, 921-926.

(20) Sakata, T.; Miyahara, Y. Noninvasive Monitoring of Transporter-Substrate Interaction at Cell Membrane. Anal. Chem. 2008, 80, $1493-1496$

(21) Rothberg, J. M.; Hinz, W.; Rearick, T. M.; Schultz, J.; Mileski, W.; Davey, M.; Leamon, J. H.; Johnson, K.; Milgrew, M. J.; Edwards, M.; Hoon, J.; Simons, J. F.; Marran, D.; Myers, J. W.; Davidson, J. F.; Branting, A.; Nobile, J. R.; Puc, B. P.; Light, D.; Clark, T. A.; Huber, M.; Branciforte, J. T.; Stoner, I. B.; Cawley, S. E.; Lyons, M.; Fu, Y.; Homer, N.; Sedova, M.; Miao, X.; Reed, B.; Sabina, J.; Feierstein, E.; Schorn, M.; Alanjary, M.; Dimalanta, E.; Dressman, D.; Kasinskas, R.; Sokolsky, T.; Fidanza, J. A.; Namsaraev, E.; McKernan, K. J.; Williams, A.; Roth, G. T.; Bustillo, J. An integrated semiconductor device enabling non-optical genome sequencing. Nature 2011, 475, $348-352$.

(22) Sakata, T.; Matsuse, Y. In Situ Electrical Monitoring of Cancer Cells Invading Vascular Endothelial Cells with Semiconductor-Based Biosensor. Genes Cells 2017, 22, 203-209.

(23) Sakata, T.; Matsumoto, S.; Nakajima, Y.; Miyahara, Y. Potential Behavior of Biochemically Modified Gold Electrode for ExtendedGate Field-Effect Transistor. Jpn. J. Appl. Phys. 2005, 44, 2860-2863.

(24) Kajisa, T.; Sakata, T. Fundamental Properties of Phenylboronic-acid-coated Gate Field-effect Transistor For Saccharide Sensing. ChemElectroChem 2014, 1, 1647-1655.

(25) Springsteen, G.; Wang, B. A Detailed Examination of Boronic Scid-diol Complexation. Tetrahedron 2002, 58, 5291-5300.

(26) Lu, Q.; Danner, E.; Waite, J. H.; Israelachvili, J. N.; Zeng, H.; Hwang, D. S. Adhesion of Mussel Foot Proteins to Different Substrate Surfaces. J. R. Soc., Interface 2013, 10, No. 20120759.

(27) Zangmeister, R. A.; Morris, T. A.; Tarlov, M. J. Characterization of Polydopamine Thin Films Deposited at Short Times by Autoxidation of Dopamine. Langmuir 2013, 29, 8619-8628.

(28) d'Ischia, M.; Napolitano, A.; Pezzella, A.; Meredith, P.; Sarna, T. Chemical and Structural Diversity in Eumelanins: Unexplored BioOptoelectronic Materials. Angew. Chem., Int. Ed. 2009, 48, 39143921.

(29) Lynge, M. E.; van der Westen, R.; Postma, A.; Stadler, B. Polydopamine-a Nature-Inspired Polymer Coating for Biomedical Science. Nanoscale 2011, 3, 4916-4928.

(30) Amiri, M.; Amali, E.; Nematollahzadeh, A.; Salehniya, H. PolyDopamine Films: Voltammetric Sensor for $\mathrm{pH}$ Monitoring. Sens. Actuators, B 2016, 228, 53-58.

(31) Hill, A. V. The Possible Effects of the Aggregation of the Molecules of Haemoglobin on Its Dissociation Curves. J. Physiol. 1910, 40, 4-7.

(32) Maue, M.; Schrader, T. A Color Sensor for Catecholamines. Angew. Chem., Int. Ed. 2005, 44, 2265-2270.

(33) Secor, K. E.; Glass, T. E. Selective Amine Recognition: Development of a Chemosensor for Dopamine and Norepinephrine. Org. Lett. 2004, 6, 3727-3730.

(34) Huang, Y.-J.; Jiang, Y.-B.; Fossey, J. S.; James, T. D.; Marken, F. Assembly of $\mathrm{N}$-Hexadecyl-Pyridinium-4-Boronic Acid Hexafluorophosphate Monolayer Films with Catechol Sensing Selectivity. J. Mater. Chem. 2010, 20, 8305-8310.

(35) Coskun, A.; Akkaya, E. U. Three-Point Recognition and Selective Fluorescence Sensing of L-Dopa. Org. Lett. 2004, 6, 31073109.

(36) Jin, S.; Li, M.; Zhu, C.; Tran, V.; Wang, B. Computer-Based De Novo Design, Synthesis, and Evaluation of Boronic Acid-Based Artificial Receptors for Selective Recognition of Dopamine. ChemBioChem 2008, 9, 1431-1438. 
(37) Kaiser, H. Die Berechnung Der Nachweisempfindlichkeit. Spectrochim. Acta 1947, 3, 40-67.

(38) Olanow, C. W.; Gauger, L. L.; Cedarbaum, J. M. Temporal Relationships between Plasma and Cerebrospinal Fluid Pharmacokinetics of Levodopa and Clinical Effect in Parkinson's Disease. Ann. Neurol. 1991, 29, 556-559.

(39) Cuche, J.-L.; Prinseau, J.; Selz, F.; Ruget, G.; Baglin, A. Plasma Free, Sulfo-and Glucuro-Conjugated Catecholamines in Uremic Patients. Kidney Int. 1986, 30, 566-572.

(40) Routh, J.; Bannow, R.; Fincham, R.; Stoll, J. Excretion of LDopa and Its Metabolites in Urine of Parkinson's Disease Patients Receiving L-Dopa Therapy. Clin. Chem. 1971, 17, 867-871.

(41) Holzman, C.; Senagore, P.; Tian, Y.; Bullen, B.; DeVos, E.; Leece, C.; Zanella, A.; Fink, G.; Rahbar, M. H.; Sapkal, A. Maternal Catecholamine Levels in Midpregnancy and Risk of Preterm Delivery. Am. J. Epidemiol. 2009, 170, 1014-1024.

(42) Zubareva, T. V.; Kiseleva, Z. Catecholamine Content of the Lacrimal Fluid of Healthy People and Glaucoma Patients. Ophthalmologica 1977, 175, 339-344. 